\title{
NON-CENTRALIZED DisTINCT L-DivERSITY
}

\author{
Chi Hong Cheong ${ }^{1}$, Dan $\mathrm{Wu}^{2}$, and Man Hon Wong ${ }^{3}$ \\ ${ }^{1,3}$ Department of Computer Science and Engineering, \\ The Chinese University of Hong Kong, Hong Kong \\ \{chcheong, mhwong\} @cse. cuhk.edu.hk \\ ${ }^{2}$ Network and Telecommunication Division \\ Guangdong Electric Power Design Institute, China \\ wudan@gedi.com.cn
}

\begin{abstract}
This paper considers the non-centralized version of privacy preserving data publishing (PPDP), which refers to generating published tables from multiple non-centralized private tables owned by different data holders. Traditional solutions to PPDP on a single centralized dataset cannot be directly applied to this problem. Even if every published table satisfies a traditional privacy preserving requirement individually, an adversary who can collect multiple published tables may be able to deduce some private information that violates the satisfied requirement. Due to privacy reasons, the data holders cannot share information with each other to cooperate on the data publishing issues. In this paper, we propose non-centralized distinct l-diversity and an algorithm to generate published tables. Our algorithm does not rely on any communications between the data holders but only collects published tables released by other data holders. Experiments on real datasets are conducted to show that the algorithm is feasible to real applications.
\end{abstract}

\section{KEYWORDS}

Non-centralized, Privacy Preserving Data Publishing, Database, Conditional Independence

\section{INTRODUCTION}

Traditional solutions to the problem of privacy preserving data publishing (PPDP), such as $k$ anonymity [1], $l$-diversity [2], and $t$-closeness [3], only guarantee that privacy is preserved if a published table is created from a centralized dataset. Recently, many researchers have shown that if there are multiple published tables created from a centralized dataset (which can be either dynamic or static), privacy may not be preserved even if every published table satisfies a certain privacy preserving requirement individually [4][5][6][7][8]. This problem is called serial data publishing (on a centralized dataset). In this paper, we consider a similar but different problem: data publishing on non-centralized datasets. The non-centralized datasets that we refer to are multiple datasets that have a common sensitive attribute with the same domain and store information about the same group of individuals, but they are owned by different data holders that cannot share information with each other due to privacy reasons. We are going to show that privacy may not be preserved even if all published tables generated from such multiple noncentralized datasets satisfy certain privacy preserving requirements for data publishing individually. It is illustrated in the following example.

Table 1 shows two private tables, namely $\operatorname{PriT}_{R}$ and $\operatorname{PriT}_{C}$, which are solely owned by the Revenue Department and the Censor Department respectively. Both $\operatorname{PriT}_{R}$ and $\operatorname{PriT}_{C}$ store income information of the same group of individuals. Some data miners may find such information useful for their manpower researches. However, due to privacy reasons or even enforced by law, private tables $\operatorname{PriT}_{R}$ and $\operatorname{PriT}_{C}$ cannot be released directly to any other parties.

DOI: $10.5121 / \mathrm{ijdms} .2012 .4201$ 
International Journal of Database Management Systems ( IJDMS ) Vol.4, No.2, April 2012

Instead, the Revenue Department and the Censor Department release two published tables, denoted as $T_{\text {age }}$ and $T_{\text {ZIP code }}$, which are created by generalizing or suppressing the attributes of

Table 1. Two private tables $\operatorname{PriT}_{R}$ and $\operatorname{PriT}_{C}$.

\begin{tabular}{|c|c|c|c|}
\hline Name & Age & Gender & Salary Class \\
\hline Alice & 60 & F & High \\
\hline Bob & 50 & M & High \\
\hline Carlo & 55 & M & High \\
\hline Diana & 50 & F & High \\
\hline Eva & 48 & F & Middle \\
\hline Fred & 43 & M & Middle \\
\hline George & 25 & M & Middle \\
\hline Helen & 37 & F & Middle \\
\hline Ivan & 57 & M & Low \\
\hline Janice & 57 & F & Low \\
\hline Kate & 18 & F & Low \\
\hline Leslie & 22 & F & Low \\
\hline
\end{tabular}

(a) Private table PriT $_{R}$

\begin{tabular}{|c|c|c|}
\hline Name & ZIP Code & Salary Class \\
\hline Alice & 25434 & High \\
\hline Bob & 27343 & High \\
\hline Carlo & 19343 & High \\
\hline Diana & 17234 & High \\
\hline Eva & 28544 & Middle \\
\hline Fred & 24453 & Middle \\
\hline George & 26211 & Middle \\
\hline Helen & 23094 & Middle \\
\hline Ivan & 29454 & Low \\
\hline Janice & 12845 & Low \\
\hline Kate & 15341 & Low \\
\hline Leslie & 22093 & Low \\
\hline
\end{tabular}

(b) Private table PriT $_{C}$

Table 2. Two published tables $T_{\text {age }}$ and $T_{Z I P \text { code }}$.

\begin{tabular}{|c|c|}
\hline Age & Salary Class \\
\hline$\geq 40$ & High \\
$\geq 40$ & High \\
$\geq 40$ & High \\
$\geq 40$ & High \\
$\geq 40$ & Middle \\
$\geq 40$ & Middle \\
$\geq 40$ & Low \\
$\geq 40$ & Low \\
\hline$<40$ & Middle \\
$<40$ & Middle \\
$<40$ & Low \\
$<40$ & Low \\
\hline
\end{tabular}

(a) Published Table $T_{\text {age }}$

\begin{tabular}{|c|c|}
\hline ZIP Code & Salary Class \\
\hline$\geq 20 \mathrm{k}$ & High \\
$\geq 20 \mathrm{k}$ & High \\
$\geq 20 \mathrm{k}$ & High \\
$\geq 20 \mathrm{k}$ & High \\
$\geq 20 \mathrm{k}$ & Middle \\
$\geq 20 \mathrm{k}$ & Middle \\
$\geq 20 \mathrm{k}$ & Low \\
$\geq 20 \mathrm{k}$ & Low \\
\hline$<20 \mathrm{k}$ & Middle \\
$<20 \mathrm{k}$ & Middle \\
$<20 \mathrm{k}$ & Low \\
$<20 \mathrm{k}$ & Low \\
\hline
\end{tabular}

(b) Published Table $T_{\text {ZIP code }}$

$\operatorname{PriT}_{R}$ and PriT $_{C}$ respectively. An example is shown in Table 2, where $T_{\text {age }}$ and $T_{Z I P}$ code individually satisfy distinct 2-diversity [2], which is a traditional privacy preserving requirement for data publishing. Even if an adversary can obtain access to either $T_{\text {age }}$ or $T_{Z I P}$ code and possess the identification information (the age and the ZIP code) of an individual (the victim), the adversary cannot deduce the exact sensitive attribute value of the victim. At best, the adversary can only narrow down the number of possible sensitive attribute values to two. However, ensuring that $T_{\text {age }}$ and $T_{Z I P}$ code satisfy distinct 2-diversity individually is not enough to preserve privacy. We will show that if an adversary can obtain access to both $T_{\text {age }}$ and $T_{Z I P}$ code, the adversary may be able to deduce some information that cannot be deduced from either $T_{\text {age }}$ or $T_{Z I P}$ code individually, and such information may cause privacy breaches. Suppose an adversary knows the following identification information of the victim.

- Both $T_{\text {age }}$ and $T_{Z I P \text { code }}$ contain a tuple that corresponds to the victim.

- The victim's age $<40$ and the victim's ZIP Code $<20 \mathrm{k}$. 
International Journal of Database Management Systems ( IJDMS ) Vol.4, No.2, April 2012

From $T_{\text {age }}$ (Table 2(a)) and the fact that the victim's age $<40$, the adversary deduces that the salary class of the victim is either "Low" or "Middle". From $T_{\text {ZIP code }}($ Table 2(b)) and the fact that the victim's ZIP code $<20 \mathrm{k}$, the adversary deduces that the salary class of the victim is either "Low" or "High". Since both $T_{\text {age }}$ and $T_{Z I P}$ code have a tuple that corresponds to the victim, the adversary can combine the above results and deduce that the salary class of the victim must be "Low". In other words, the adversary can deduce the exact sensitive value of the victim. Hence, 2-diversity is violated even if both $T_{\text {age }}$ and $T_{\text {ZIP code }}$ are 2-diverse tables individually.

Table 3. The centralized table PriT $_{R, C}$ (for centralized data publishing only).

\begin{tabular}{|c|c|c|c|c|}
\hline Name & Age & Gender & ZIP Code & Salary Class \\
\hline Alice & 60 & F & 25434 & High \\
\hline Bob & 50 & M & 27343 & High \\
\hline Carlo & 55 & M & 19343 & High \\
\hline Diana & 50 & F & 17234 & High \\
\hline Eva & 48 & F & 28544 & Middle \\
\hline Fred & 43 & M & 24453 & Middle \\
\hline George & 25 & M & 26211 & Middle \\
\hline Helen & 37 & F & 23094 & Middle \\
\hline Ivan & 57 & M & 29454 & Low \\
\hline Janice & 57 & F & 12845 & Low \\
\hline Kate & 18 & F & 15341 & Low \\
\hline Leslie & 22 & F & 22093 & Low \\
\hline
\end{tabular}

Table 4. A published table $T_{\text {age, ZIP code }}$ (for centralized data publishing only).

\begin{tabular}{|c|c|c|}
\hline Age & ZIP Code & Salary Class \\
\hline$\geq 40$ & $\geq 20 \mathrm{k}$ & High \\
$\geq 40$ & $\geq 20 \mathrm{k}$ & High \\
$\geq 40$ & $\geq 20 \mathrm{k}$ & Middle \\
$\geq 40$ & $\geq 20 \mathrm{k}$ & Middle \\
$\geq 40$ & $\geq 20 \mathrm{k}$ & Low \\
\hline$\geq 40$ & $<20 \mathrm{k}$ & High \\
$\geq 40$ & $<20 \mathrm{k}$ & High \\
$\geq 40$ & $<20 \mathrm{k}$ & Low \\
\hline$<40$ & $\geq 20 \mathrm{k}$ & Middle \\
$<40$ & $\geq 20 \mathrm{k}$ & Middle \\
$<40$ & $\geq 20 \mathrm{k}$ & Low \\
\hline$<40$ & $<20 \mathrm{k}$ & Low \\
\hline
\end{tabular}

The Revenue Department and the Censor Department not only need to ensure that their published tables, $T_{\text {age }}$ and $T_{Z I P}$ code, satisfy a certain privacy preserving requirement individually, but also have to ensure that no privacy breaches will occur if both published tables are obtained by an adversary. The solution is trivial if the Revenue Department and the Censor Department can share their private tables, $\operatorname{PriT}_{R}$ and $P r i T_{C}$, with each other. It is because they can first combine $\operatorname{PriT}_{R}$ and $\operatorname{PriT}_{C}$ together to form a centralized table $\operatorname{PriT}_{R, C}$ as shown in Table 3 by matching the name attributes of the two private tables. Then, they can construct published table $T_{\text {age, ZIP code }}$ as shown in Table 4 by generalizing the age and the ZIP code attributes of the centralized table PriT $T_{R, C}$. Both $T_{\text {age }}$ and $T_{Z I P \text { code }}$ can be obtained by suppressing an attribute in $T_{\text {age, ZIP code. }}$. If $T_{\text {age, }}$ ZIP code satisfies a certain privacy preserving requirement, releasing both $T_{a g e}$ and $T_{Z I P \text { code }}$ does not cause privacy breaches. It is because the private information deduced from an adversary who can 
obtain access to both $T_{\text {age }}$ and $T_{Z I P \text { code }}$ must be no more than the private information stored in $T_{\text {age, }}$ ZIP code. However, in our example, $T_{\text {age, ZIP code }}$ (Table 4) is only a 1-diverse table. Hence, the Revenue Department and the Censor Department know that the adversary may be able to deduce the exact sensitive attribute value of an individual if the adversary can obtain access to both $T_{\text {age }}$ and $T_{Z I P \text { code }}$. Therefore, they will not release $T_{\text {age }}$ and $T_{\text {ZIP code }}$ together and thus privacy breaches are avoided.

Unfortunately, the above solution may not be applicable in practice. It is because due to privacy reasons or even enforced by law, the Revenue Department and the Censor Department cannot share their private tables with each other (though both departments belong to the government). It follows that no one can precisely construct $P r i T_{R, C}$ and generate $T_{\text {age, ZIP code. Hence, the above }}$ solution cannot be applied when such a restriction is enforced. With such a restriction, this problem becomes non-centralized data publishing. The solution to this problem is not trivial since the Revenue Department and the Censor Department cannot share information with each other, but they still have to generate $T_{a g e}$ and $T_{Z I P}$ code that do not cause privacy breach even if there is an adversary who can obtain access to both $T_{\text {age }}$ and $T_{\text {ZIP code }}$.

The objective of this paper is to present a way to release published tables that will not cause privacy breaches in non-centralized environment. Firstly, we propose two approaches for a data holder to determine the private information that will be deduced by an adversary who can get access to all non-centralized published tables if the data holder releases a published table. Since there may be correlations among the attributes in different published tables, two approaches are needed. The first approach is for the scenario that the adversary knows such correlations. This approach determines the private information deduced by such an adversary by considering how the adversary utilizes the correlations to deduce the private information. The second approach is for the scenario that the adversary does not know such correlations. This approach determines the private information deduced by such an adversary by considering how the adversary makes a conditional independent assumption to deduce private information. For both approaches, the data holder does not need to communicate with other data holders. Instead, the data holder only needs to collect published tables generated by other data holders. Secondly, we propose how to adopt distinct $l$-diversity [2], which is a privacy preserving requirement for centralized data publishing, to non-centralized data publishing. It is so called non-centralized distinct $l$-diversity. Thirdly, we propose an algorithm for a data holder to generate published tables that satisfy non-centralized distinct $l$-diversity. Our algorithm is modified from Incognito [13], which is an efficient and widely-used algorithm for (centralized) data publishing. Theorems are presented to show the correctness of the proposed algorithm. Experiments on real datasets are conducted to show that the proposed algorithm is feasible to real applications.

This paper is organized as follows. Section 2 presents the related work. Section 3 presents the table models used in this paper. Section 4 discusses the private information deduced from multiple published tables in a non-centralized environment. Section 5 presents the noncentralized version of distinct $l$-diversity and an algorithm for non-centralized PPDP. Section 6 presents the experiment results. Finally, conclusions are presented in Section 7.

\section{RELATED WORK}

This paper focuses on data publishing on non-centralized datasets. There has been extensive research in PPDP, such as $k$-anonymity [1], $l$-diversity [2], and $t$-closeness [3]. However, they focus on the cases for a single centralized dataset and a single published table. In the previous section, we have shown that these solutions cannot be directly applied to the cases for multiple published tables.

Recently, the problem of serial data publishing has attracted a lot of attentions. These research works can be classified into serial data publishing on a dynamic dataset and serial data publishing on a static dataset. Most of the works focus on the cases for a dynamic dataset (or multiple 
instances of data), which includes an incremental dataset [4][5] (i.e. allowing insertions of new tuples), a dataset with insertions and deletions [6], a dataset with insertions, deletions, and updates [7], a dataset with sensitive values that change over time [8]. Some other works focus on the cases for a static dataset, such as [9]. Nevertheless, all such works cannot be applied to our problem. It is because they require the data holder to possess a dataset or a series of dataset instances that contains all information. However, in the non-centralized datasets problem, no one possesses all information.

We propose a statistical approach to determine the private information deduced by an adversary and the results are stored in a probabilistic table. Many previous works also use probabilistic approach [10] or deal with probabilistic databases [11][12].

Our proposed algorithm that generates published tables is modified from Incognito [13]. Similar to many previous works [1][14][15][16], published tables are generated by generalizing and removing the quasi-identifier attributes of the private table, while remaining the sensitive attribute unchanged.

In the non-centralized data publishing problem, no one (neither the data holders nor the adversaries) is able to combine all the private/published tables and deduce the actual correlations among the quasi-identifier attributes in different private/published tables. Without such correlations, even if an adversary can get access to two or more published tables, the adversary can only deduce information stored in the tables individually, but not the "combined" information reflected by these tables. Nevertheless, the adversary can make the conditional independence assumption, which is not uncommon in database research works [17][18][19][20] and other research works [21], such as Bayesian analysis [22][23], to deduce the "combined" information reflected by the published tables.

This paper focuses on PPDP, which focuses on how to publish data. There is another related area called privacy preserving data mining (PPDM), which focuses on how to perform data mining on the data that is modified to preserve privacy [24][25][26].

\section{TABLE MODELS}

In this section, we present the private table model (Section 3.1) and the published table model (Section 3.2).

\subsection{Private Table}

A private table stores private information about a group of individuals. It is solely owned by a data holder and is never released to other parties directly. In this paper, we focus on the case that there are multiple non-centralized private tables that store information about the same group of individuals. For example, Table 1 shows two private tables, namely $\operatorname{PriT}_{R}$ and $\operatorname{PriT}_{C}$, which are solely owned by the Revenue Department and the Censor Department respectively. Both PriT $_{R}$ and $\operatorname{PriT}_{C}$ store income information about a group of individuals. A tuple in the private table corresponds to an individual. A private table consists of three sets of attributes:

- Explicit-identifier attributes. Each of them can uniquely identify an individual in the private table, such as the name.

- Quasi-identifier attributes [1]. It is a set of attributes that may uniquely identify an individual in the private table when some of such attributes are considered together and linked to an external dataset, such as the age and the gender.

- A sensitive attribute. It stores sensitive information about an individual, such as the salary class. In this paper, we consider the private tables that share a common sensitive attribute and store information about the same group of individuals. 
International Journal of Database Management Systems ( IJDMS ) Vol.4, No.2, April 2012

\subsection{Published Table}

Some parties, say data miners, may find the information stored in the private table useful for research or other purposes. However, due to privacy reasons or even enforced by law, the data holder cannot release the private table to the data miners directly. Hence, the data holder needs to generate a table with less information and release it to the data miners instead. Such a table is called a published table. A popular approach to construct a published table from a private table is to suppress the explicit identifier attributes, generalize or suppress the quasi-identifier attributes, and retain the sensitive attribute of the private table [1][14][15][16]. For example, $T_{\text {age }}$ and $T_{Z I P}$ code in Table 2 are two published tables for private tables $\operatorname{PriT}_{R}$ and $\operatorname{PriT}_{C}$ respectively. This paper considers non-centralized data publishing, which refers to generating published tables from multiple non-centralized private tables, where each private table has a published table. Let $n$ be the number of private tables. Then, there will be $n$ published tables. Let $T_{i}$ be the published table for the private table $\operatorname{PriT}_{i}$, where $1 \leq i \leq n$. A published table $T_{i}$ consists of two sets of attributes:

- Quasi-identifier attributes. Each of them is generalized from a quasi-identifier attribute in the corresponding private table. Some quasi-identifier attributes may be suppressed and thus not all quasi-identifier attributes in the private table appear in the published table. Let $m$ be the number of quasi-identifier attributes among all published tables. The quasi-identifier attributes are denoted as $A_{i}$ for $1 \leq i \leq m$. In this paper, duplicate quasiidentifier attributes among the published tables are discarded. A group of tuples with the same quasi-identifier attribute values form an equivalence class (EC) (or a QI-group). For example, $T_{\text {age }}$ in Table 2(a) has one quasi-identifier attribute age and two ECs, namely $E C_{\text {age }} \geq 40$ and $E C_{\text {age }}<40$.

- A sensitive attribute. All published tables share a common sensitive attribute $S$, which is the same as the sensitive attribute shared by all private tables.

\section{Private Information Deduced from Multiple Published Tables}

In this section, we discuss the private information deduced by an adversary who can obtain access to multiple published tables for non-centralized data publishing. Data holders have to know what private information can be deduced by such an adversary in order to generate published tables that do not cause privacy breaches. Section 4.1 shows that some private information can be deduced by simple counting on each published table individually. Section 4.2 shows that more private information can be deduced by considering multiple published tables together with (Section 4.2.1) or without (Section 4.2.2) knowing the correlation among the quasi-identifier attributes. Section 4.3 shows that the deduced private information can be systematically stored in a probabilistic table, which is needed for our proposed algorithm to generate non-centralized published tables.

\subsection{Private Information Deduced by Simple Counting on Each Published Tables}

Consider a victim who has a tuple in both published tables $T_{\text {age }}$ and $T_{\text {ZIP code }}$ depicted in Table 2. Let $h, m$, and $l$ be the events that the salary classes of the victim are "High", "Middle", and "Low" respectively. Let $\mathrm{P}(H), \mathrm{P}(M)$, and $\mathrm{P}(L)$ be the probabilities that the salary classes of the victim are "High", "Middle", and "Low" respectively. We assume that the private tables are consistent and hence $\mathrm{P}(H)$ can be computed by counting the number of tuples with sensitive attribute values = "High" in either $T_{\text {age }}$ or $T_{Z I P \text { code }}$. In our example, there are four out of 12 tuples with values equal to "High" in each of $T_{\text {age }}$ and $T_{Z I P}$ code, which store information about the same group of individuals and the sensitive attribute values are the same. Hence, we have $\mathrm{P}(H)=4 / 12=0.333$. Similarly, we have $\mathrm{P}(M)=\mathrm{P}(L)=0.333$.

Consider an adversary who (1) can obtain access to both $T_{\text {age }}$ and $T_{Z I P \text { code }},(2)$ knows that there is a tuple in both $T_{\text {age }}$ and $T_{Z I P \text { code }}$ that corresponds to the victim, and (3) knows that the victim's age $\geq$ 40 and the victim's ZIP code $\geq 20 \mathrm{k}$. The adversary can deduce some private information, in terms of conditional probabilities, about the victim from each of $T_{\text {age }}$ and $T_{Z I P}$ code individually. First we 
International Journal of Database Management Systems ( IJDMS ) Vol.4, No.2, April 2012

consider $T_{\text {age }}$. Since the victim's age $\geq 40$, the tuple that corresponds to the victim must be one of the first eight tuples. Among these eight tuples, four of them have the sensitive attribute value = "High". Hence, the conditional probability that the salary class of the victim is "High" given that the victim's age $\geq 40$ is $4 / 8=0.5$. Such a conditional probability is denoted as $\mathrm{P}(H \mid A \geq 40)$, where $A$ be the age of the victim. Similarly, the adversary can deduce conditional probabilities $\mathrm{P}(M \mid A \geq$ $40)=0.25$ and $\mathrm{P}(L \mid A \geq 40)=0.25$ from $T_{\text {age }}$. Next we consider $T_{Z I P \text { code }}$. Let $Z$ be the ZIP code of the victim. Similarly, the adversary can deduce $\mathrm{P}(H \mid Z \geq 20 \mathrm{k})=0.25, \mathrm{P}(M \mid Z \geq 20 \mathrm{k})=0.5$, and $\mathrm{P}(L \mid Z \geq 20 \mathrm{k})=0.25$ from $T_{Z I P \text { code }}$.

\subsection{Private Information Deduced from Multiple Published Tables}

As mentioned in Section 1, although published tables $T_{\text {age }}$ and $T_{Z I P}$ code (Table 2) satisfy the 2diversity requirement individually, an adversary who can obtain access to both $T_{\text {age }}$ and $T_{\text {ZIP code }}$ may be able to deduce further private information that violates such a requirement. We propose some approaches to determine such further private information deduced by the adversary. The proposed approach can be used to verify whether releasing a non-centralized published table cause privacy breaches, which is an important step for our proposed algorithm to generate noncentralized published tables.

In Section 4.1, we presented the conditional probabilities deduced from each of $T_{\text {age }}$ and $T_{\text {ZIP code }}$ by the simple counting approach. The conditions of such conditional probabilities involve either $A$ or $Z$, but not both. As an example, the conditional probabilities deduced in the previous subsection are $\mathrm{P}(H \mid A \geq 40)$ and $\mathrm{P}(H \mid Z \geq 20 \mathrm{k})$. However, the adversary actually wants to compute $\mathrm{P}(H \mid A \geq 40, Z \geq 20 \mathrm{k})$, which is the conditional probability that the sensitive attribute value of the victim is high given that $A \geq 40$ and $Z \geq 20 \mathrm{k}$. It is because the adversary knows the age and the ZIP code of the victim and is able to obtain access to both $T_{\text {age }}$ and $T_{Z I P}$ code.

Although the age and the ZIP code attributes are in different private tables hold by different data holders, it is not surprising that there is a correlation between the two attributes. For example, a city may have more elder people than younger people. Hence, the probability that an individual who lives in such a city is an elderly is higher. There are two ways to compute $\mathrm{P}(H \mid A \geq 40, Z \geq 20 \mathrm{k})$. The first way is for the adversary who does not know the QI attribute correlation. Such an adversary can make the conditional independent assumption to compute $\mathrm{P}(H \mid A \geq 40, Z \geq 20 \mathrm{k})$. The second way is for the adversary who does not know the QI attribute correlation. Such an adversary can use a statistical approach to compute $\mathrm{P}(H \mid A \geq 40, Z \geq 20 \mathrm{k})$. In the rest of this subsection, we will discuss these two ways to compute $\mathrm{P}(H \mid A \geq 40, Z \geq 20 \mathrm{k})$.

\subsubsection{Without Knowing the Quasi-Identifier Attribute Correlation}

Consider an adversary who does not know the correlation between the age and the ZIP code attributes. It seems that the adversary can compute the $\mathrm{P}(H \mid A \geq 40, Z \geq 20 \mathrm{k})$ value by assuming that the age and the ZIP code attributes are independent, and then use the independence property $\mathrm{P}(A \geq 40, Z \geq 20 \mathrm{k})=\mathrm{P}(H \mid A \geq 40) \cdot \mathrm{P}(Z \geq 20 \mathrm{k})$. Unfortunately, such an assumption cannot be made. It is because the knowledge about the ZIP code of an individual may be updated by knowing the age of the individual. It is illustrated in the following example. Suppose the adversary does not know the age of the individual. In $T_{Z I P \text { code }}$, there are 12 tuples and eight of them have ZIP code $\geq 20 \mathrm{k}$. Hence, the probability that the individual's ZIP code $\geq 20 \mathrm{k}$ is $2 / 3$. Such a probability is denoted as $p$. On the other hand, suppose the adversary knows that the individual's age $<40$. From $T_{\text {age }}$, the adversary knows the individual's tuple must be one of the last four tuples. Hence, the salary class of the individual is either "Low" or "Middle" with equal chance. With such information, the adversary can eliminate the tuples with sensitive attribute value $=$ "High" in $T_{\text {ZIP code }}$. Among the remaining eight tuples, six of them have ZIP code $\geq 20 \mathrm{k}$. Hence, the adversary now has $p=3 / 4$. In other words, by knowing the victim's age $<40$, the value of $p$ is updated from $2 / 3$ to $3 / 4$. This counter example explains why the adversary cannot assume that the age and the ZIP code attributes are independent. 
International Journal of Database Management Systems ( IJDMS ) Vol.4, No.2, April 2012

In fact, to compute $\mathrm{P}(H \mid A \geq 40, Z \geq 20 \mathrm{k})$, the adversary who does not know the QI attribute correlation can make the following assumption: the age and ZIP code attributes are conditionally independent given the value of the salary class attribute. In probability theory, conditional independence for events $E_{1}$ and $E_{2}$ is defined as follows: $E_{1}$ and $E_{2}$ are two conditionally independent events given event $G$ if and only if $\mathrm{P}\left(E_{1}, E_{2} \mid G\right)=\mathrm{P}\left(E_{l} \mid G\right) \cdot \mathrm{P}\left(E_{2} \mid G\right)$.

Here we use an example to illustrate how to make the conditional independence assumption in our problem. If the salary class of the individual is given in the first place, the knowledge about the ZIP code of an individual is independent of the knowledge of the age of the individual. In other words, knowing the ZIP code (or the age) of the individual will not update the knowledge about the age (or the ZIP code) of the individual. It is illustrated in the following example. Suppose the information that the salary class of the victim is either "Low" or "Middle" with equal chance is given in the first place. With such information, the adversary has $p=3 / 4$ already even if we do not know the individual's age and only consider $T_{Z I P}$ code. Hence, even if the adversary now knows that the individual's age $<40$, the value of $p$ will not be updated. This is also true if the salary class of the victim equals to other value(s). It is because the salary class attribute is the only connection between the age and the ZIP code attributes if the adversary does not know the QI attribute correlation. By knowing the age attribute, one can update the knowledge about the salary class attribute, which can be used to update the knowledge about the ZIP code attribute. If such an updated knowledge about the salary class attribute is given in the first place, it will update the knowledge about the ZIP code of the individual regardless of the knowledge about the age of the individual.

$$
\begin{aligned}
& \frac{P(H, A \geq 40, Z \geq 20 k)}{P(A \geq 40, Z \geq 20 k)} \\
& \frac{P(H, A \geq 40, Z \geq 20 k)}{P(H, A \geq 40, Z \geq 20 k)+P(M, A \geq 40, Z \geq 20 k)+P(L, A \geq 40, Z \geq 20 k)} \\
& \frac{P(A \geq 40, Z \geq 20 k \mid H) \cdot P(H)}{P(A \geq 40, Z \geq 20 k \mid H) \cdot P(H)+P(A \geq 40, Z \geq 20 k \mid M) \cdot P(M)+P(A \geq 40, Z \geq 20 k \mid L) \cdot P(L)} \\
& \frac{P(A \geq 40 \mid H) \cdot P(Z \geq 20 k \mid H) \cdot P(H)}{P(A \geq 40 \mid H) \cdot P(Z \geq 20 k \mid H) \cdot P(H)+P(A \geq 40 \mid M) \cdot P(Z \geq 20 k \mid M) \cdot P(M)+P(A \geq 40 \mid L) \cdot P(Z \geq 20 k \mid L) \cdot P(L)}
\end{aligned}
$$

With such an assumption, the adversary can compute $\mathrm{P}(H \mid A \geq 40, Z \geq 20 \mathrm{k})$ as follows.

- By the definition of conditional probability, $\mathrm{P}(H \mid A \geq 40, Z \geq 20 \mathrm{k})$ can be expressed as Equation (1) .

- The salary class has only three possible values, by the law of alternatives, Equation (1) can be expressed as Equation (2).

- By the product rule of conditional probabilities, Equation (2) can be expressed as (3).

- By the definition of conditional independence, Equation (3) can be expressed as Equation (4).

- By simple counting on $T_{\text {age }}$ and $T_{Z I P}$ code individually, the adversary can deduce $\mathrm{P}(A \geq 40 \mid H)=0.5, \mathrm{P}(Z \geq 20 \mathrm{k} \mid H)=0.25, \mathrm{P}(H)=0.333, \mathrm{P}(A \geq 40 \mid M)=0.25$, $\mathrm{P}(Z \geq 20 \mathrm{k} \mid M)=0.5, \mathrm{P}(M)=0.333, \mathrm{P}(A \geq 40 \mid L)=0.25, \mathrm{P}(Z \geq 20 \mathrm{k} \mid L)=0.25$, and $\mathrm{P}(L)=0.333$. By substituting these values into the Equation (4), the adversary can obtain: $\mathrm{P}(H \mid A \geq 40, Z \geq 20 \mathrm{k})=0.4$.

Similarly, the adversary can also calculate the conditional probabilities for other sensitive attribute values: $\mathrm{P}(M \mid A \geq 40, Z \geq 20 \mathrm{k})=0.4$ and $\mathrm{P}(L \mid A \geq 40, Z \geq 20 \mathrm{k})=0.2$.

In general, suppose there are $n$ published tables, namely $T_{1}, \ldots, T_{n}$, which are generated from $n$ private tables $\operatorname{PriT}_{1}, \ldots, \operatorname{PriT}_{n}$ respectively. Further suppose there are $m$ quasi-identifier attributes, namely $A_{l}, \ldots, A_{m}$, which are distributed among these $n$ published tables. The $n$ published tables can be formulated as:

$$
T_{1}=\left\{A_{1}, \ldots, A_{r_{1}}, S\right\}, T_{2}=\left\{A_{r_{1}+1}, \ldots, A_{r_{2}}, S\right\}, \ldots, T_{n}=\left\{A_{r_{n-1}+1}, \ldots, A_{m}, S\right\},
$$




$$
\text { where } 1 \leq r_{1}<r_{2}<\ldots<r_{n-1}<m \text {. }
$$

The conditional independence assumption for such a general case is stated as follows.

Assumption 1. The quasi-identifier attributes among different published tables, i.e. $A_{1}, \ldots, A_{r_{1}}$ in $T_{1}, A_{r_{1}+1}, \ldots, A_{r_{2}}$ in $T_{2}, \ldots$, and $A_{r_{n-1}+1}, \ldots, A_{m}$ in $T_{n}$, are conditionally independent given the value of sensitive attribute $S$.

Assumption 1 is needed by the adversary who does not know the QI attribute correlation to deduce private information from multiple published tables by considered them together. Note that if the adversary knows the QI attribute correlation, the adversary does not need this assumption to deduce the required private information (see the next part of this subsection).

The idea of Assumption 1 is that if the value of $S$ is given in the first place, the knowledge about the $A_{l}, \ldots, A_{m}$ values of an individual are independent to each other. In other words, knowing the $A_{i}$ value of the individual will not update the knowledge about the $A_{j}$ value of the individual for 1 $\leq i \neq j \leq m$. In probability theory, conditional independence for events $E_{l}, \ldots, E_{m}$ is defined as follows.

Definition 1. (Conditional independence for $m$ events) $E_{l}, \ldots, E_{m}$ are conditionally independent events given event $G$ if and only if $P\left(E_{1}, \ldots, E_{m} \mid G\right)=\prod_{i=1}^{m} P\left(E_{i} \mid G\right)$ for $m \geq 2$.

Consider the worst case that the adversary can obtain access to all $n$ published tables and know the values of all $m$ quasi-identifier attributes for the victim. Let $S_{k}$ be a possible value of $S$ for $1 \leq$ $k \leq s$.

Here shows that the adversary is able to compute $\mathrm{P}\left(S_{k} \mid A_{l} \geq v_{l}, \ldots, A_{m} \geq v_{m}\right)$, which is conditional probability that the sensitive attribute value of the victim is $S_{k}$ given that $A_{l} \geq v_{l}, \ldots, A_{m} \geq v_{m}$.

$\mathrm{P}\left(S_{k} \mid A_{l} \geq v_{l}, \ldots, A_{m} \geq v_{m}\right)$ can be expressed as an expression containing terms that can be calculated by simple counting on the published tables (such an expression is the general case for Equation (4). By the definition of conditional probability, it can be expressed as

$$
\frac{P\left(S_{k}, A_{1} \geq v_{1}, \ldots, A_{m} \geq v_{m}\right)}{P\left(A_{1} \geq v_{1}, \ldots, A_{m} \geq v_{m}\right)}
$$

By the law of alternatives, the above can be expressed as

$$
\frac{P\left(S_{k}, A_{1} \geq v_{1}, \ldots, A_{m} \geq v_{m}\right)}{\sum_{j=1}^{S} P\left(S_{j}, A_{1} \geq v_{1}, \ldots, A_{m} \geq v_{m}\right)}
$$

By the product rule of conditional probabilities, the above can be expressed as

$$
\frac{P\left(A_{1} \geq v_{1}, \ldots, A_{m} \geq v_{m} \mid S_{k}\right) \cdot P\left(S_{k}\right)}{\sum_{j=1}^{S} P\left(A_{1} \geq v_{1}, \ldots, A_{m} \geq v_{m} \mid S_{j}\right) \cdot P\left(S_{j}\right)}
$$

By Assumption 1 and Definition 1, the above can be expressed as

$$
\frac{\left(\prod_{i=1}^{n} p_{i, k}\right) \cdot P\left(S_{k}\right)}{\sum_{j=1}^{S}\left(\left(\prod_{i=1}^{n} p_{i, j}\right) \cdot P\left(S_{j}\right)\right)}
$$

where $\quad p_{1, k}=P\left(A_{1} \geq v_{1}, \ldots, A_{r_{1}} \geq v_{r_{1}} \mid S_{k}\right), \quad p_{2, k}=P\left(A_{r_{1}+1} \geq v_{r_{1}+1}, \ldots, A_{r_{2}} \geq v_{r_{2}} \mid S_{k}\right)$, $\ldots, p_{n, k}=P\left(A_{r_{n-1}+1} \geq v_{r_{n-1}+1}, \ldots, A_{m} \geq v_{m} \mid S_{k}\right)$, and $v_{i}$ is a value in the domain of $A_{i}$ for $1 \leq i \leq m$. (Recall that $m$ is the number of QI attributes, $n$ is the number of published tables, and $s$ is the number of possible sensitive values.)

All the terms in Equation (5) can be deduced easily. As described earlier, the probabilities $\mathrm{P}\left(S_{1}\right)$, $\ldots, \mathrm{P}\left(S_{s}\right)$ can be calculated by simple counting on any one of $T_{1}, \ldots, T_{n}$; the conditional probabilities $p_{l, k}, \ldots p_{n, k}$ can be calculated by simple counting on each of $T_{1}, \ldots, T_{n}$ respectively. Therefore, the adversary is able to compute $\mathrm{P}\left(S_{k} \mid A_{l} \geq v_{l}, \ldots, A_{m} \geq v_{m}\right)$. 
International Journal of Database Management Systems ( IJDMS ) Vol.4, No.2, April 2012

Similarly, the adversary can also calculate conditional probabilities from $\mathrm{P}\left(S_{k} \mid A_{1} \geq v_{1}, \ldots, A_{m-l} \geq v_{m-1}, A_{m}<v_{m}\right)$ to $\mathrm{P}\left(S_{k} \mid A_{1}<v_{1}, \ldots, A_{m}<v_{m}\right)$.

Table 5. A published table $T_{\text {age, ZIP code }}$ (for centralized data publishing only).

\begin{tabular}{|c|c|c|}
\hline Age & Salary Class & $\begin{array}{c}\text { Number of } \\
\text { Tuples }\end{array}$ \\
\hline \multirow{3}{*}{$\geq 40$} & High & 3 \\
& Middle & 3 \\
& Low & 4 \\
\hline \multirow{3}{*}{$<40$} & High & 4 \\
& Middle & 4 \\
& Low & 3 \\
\hline
\end{tabular}

(a) Published table $T_{\text {age }}^{\text {', }}$

\begin{tabular}{|c|c|c|}
\hline ZIP Code & Salary Class & $\begin{array}{c}\text { Number of } \\
\text { Tuples }\end{array}$ \\
\hline \multirow{3}{*}{$\geq 20 \mathrm{k}$} & High & 4 \\
& Middle & 3 \\
& Low & 2 \\
\hline \multirow{3}{*}{$<20 \mathrm{k}$} & High & 3 \\
& Middle & 4 \\
& Low & 5 \\
\hline
\end{tabular}

(b) Published table $T^{\prime}$ 'ZIP code

\subsubsection{Knowing the Quasi-Identifier Attribute Correlation}

Table 5 shows two published tables $T_{a g e}^{\prime}$ and $T_{\text {ZIP code }}^{\prime}$ with different presentations such that they store the number of tuples having each sensitive value for each EC. Such a presentation is more convenient to illustrate our idea presented in this subsection.

Consider an adversary who can get access to $T_{\text {age }}^{\prime}$ and $T_{\text {ZIP code }}^{\prime}$ and knows the correlation between the age and the ZIP code attributes. Such a correlation can be expressed in a probability equation, e.g. $\mathrm{P}(A \geq 40 \mathrm{l} Z \geq 20 \mathrm{k})=4 / 9$. We assume that such a correlation is consistent with the private tables and the published tables.

Let PriT $T_{\text {age }}^{\prime}$ and PriT ${ }_{\text {ZIP code }}^{\prime}$ be the private tables of $T_{\text {age }}^{\prime}$ and $T_{\text {ZIP code }}^{\prime}$ respectively. Since no one can get access to both $P r i T_{a g e}^{\prime}$ and PriT' ${ }_{Z I P}$ code, no one can construct a table that precisely combines the information about age, ZIP code, and salary in both PriT' ${ }_{\text {age }}$ and PriT $_{\text {ZIP code. Such a }}^{\prime}$ table is denoted as $T_{\text {age, }}^{\prime}$ ZIP code and it is an imaginary table only (it cannot be constructed precisely). Nevertheless, the adversary can still deduce the following information about the imaginary table.

- There are four tuples in $E C_{A \geq 40, Z \geq 20 k}$ in the imaginary table. It is because there are nine tuples in $E C_{Z \geq 20 k}$ in $T_{Z I P}^{\prime}$ code and $\mathrm{P}(A \geq 40 \mid Z \geq 20 \mathrm{k})=4 / 9$.

- The maximum number of tuples in $E C_{A \geq 40, Z \geq 20 k}$ in the imaginary table having sensitive value "High" is three. It is because such a number is the minimum of the number of tuples in $E C_{A \geq 40}$ in $T_{\text {age }}^{\prime}$ and the number of tuples in $E C_{Z \geq 20 k}$ in $T_{Z I P}^{\prime}$ code having sensitive value "High", which are three and four respectively.

- Similarly, the maximum numbers of tuples in $E C_{A \geq 40, Z \geq 20 k}$ in the imaginary table having sensitive value "Middle" and "Low" are three and two respectively.

From the above information, although the adversary cannot construct the imaginary table precisely, the adversary can compute conditional probabilities $\mathrm{P}(H \mid A \geq 40, Z \geq 20 \mathrm{k})$, $\mathrm{P}(M \mid A \geq 40, Z \geq 20 \mathrm{k})$, and $\mathrm{P}(L \mid A \geq 40, Z \geq 20 \mathrm{k})$ as follows.

The adversary first constructs all possible instances of the imaginary table. Let $h, m$, and $l$ be the number of tuples in an imaginary table instance having sensitive value of "High", "Middle", and "Low" respectively.

The adversary can write a simple program to find out all possible values of $h, m$, and $l$. Table 6 shows such possible values for our example. We observe that there are 10 possible imaginary table instances with equal chances. For the first instance, there are three out of four tuples in $E C_{A}$ $\geq 40, Z \geq 20 k$ with sensitive value "High". The value of $\mathrm{P}(H \mid A \geq 40, Z \geq 20 \mathrm{k})$ can be computed by considering all instances as follows: 
International Journal of Database Management Systems ( IJDMS ) Vol.4, No.2, April 2012

$$
P(H \mid A \geq 40, Z \geq 20 k)=\frac{1}{10} \cdot\left(\frac{3}{4}+\frac{3}{4}+\frac{2}{4}+\frac{2}{4}+\frac{2}{4}+\frac{1}{4}+\frac{1}{4}+\frac{1}{4}\right)=\frac{3}{8}
$$

Similarly, we have $\mathrm{P}(M \mid A \geq 40, Z \geq 20 \mathrm{k})=3 / 8$ and $\mathrm{P}(M \mid A \geq 40, Z \geq 20 \mathrm{k})=1 / 4$.

Table 6 . The possible values of $h, m$, and $l$ for all ten possible imaginary table instances.

\begin{tabular}{|c|c|c|c|}
\hline Instance & $\boldsymbol{h}$ & $\boldsymbol{m}$ & $\boldsymbol{l}$ \\
\hline 1 & 3 & 1 & 0 \\
\hline 2 & 3 & 0 & 1 \\
\hline 3 & 2 & 2 & 0 \\
\hline 4 & 2 & 1 & 1 \\
\hline 5 & 2 & 0 & 2 \\
\hline 6 & 1 & 3 & 0 \\
\hline 7 & 1 & 2 & 1 \\
\hline 8 & 1 & 1 & 2 \\
\hline 9 & 0 & 3 & 1 \\
\hline 10 & 0 & 2 & 2 \\
\hline
\end{tabular}

Table 7. Probabilistic table $P T_{\text {age,ZIP code }}$.

\begin{tabular}{|c|c|c|c|}
\hline Age & ZIP Code & Salary Class & Probability \\
\hline$\geq 40$ & $\geq 20 \mathrm{k}$ & High & 0.4 \\
$\geq 40$ & $\geq 20 \mathrm{k}$ & Middle & 0.4 \\
$\geq 40$ & $\geq 20 \mathrm{k}$ & Low & 0.2 \\
\hline$\geq 40$ & $<20 \mathrm{k}$ & High & 0.667 \\
$\geq 40$ & $<20 \mathrm{k}$ & Middle & 0 \\
$\geq 40$ & $<20 \mathrm{k}$ & Low & 0.333 \\
\hline$<40$ & $\geq 20 \mathrm{k}$ & High & 0 \\
$<40$ & $\geq 20 \mathrm{k}$ & Middle & 0.667 \\
$<40$ & $\geq 20 \mathrm{k}$ & Low & 0.333 \\
\hline$<40$ & $<20 \mathrm{k}$ & High & 0 \\
$<40$ & $<20 \mathrm{k}$ & Middle & 0 \\
$<40$ & $<20 \mathrm{k}$ & Low & 1 \\
\hline
\end{tabular}

It may not be possible for a data holder to know what the QI attribute correlation will be obtained by an adversary. Nevertheless, the data holder can still use the above equation to make the published table satisfy non-centralized l-diversity regardless of the QI attribute correlation possessed by the adversary. More details will be explained in the next subsection.

\subsection{Probabilistic Table}

The conditional probabilities computed in the previous subsections can be stored systematically in a table called a probabilistic table, which allows data holders to estimate the information deduced by an adversary in order to verify whether releasing a published table will cause privacy breaches. A probabilistic table for $n$ published tables is denoted as $P T_{1 \sim n}$. It consists of three sets of attributes:

- Quasi-identifier attributes. The quasi-identifier attributes of all previous published tables, i.e., $A_{l}, \ldots, A_{m}$. Same as an equivalence class of a published table, an equivalence class of a probabilistic table is defined as a collection of tuples with the same quasi-identifier attribute values. 
- A sensitive attribute. It is the common sensitive attribute of all previous published tables. Let $S$ be the number of possible sensitive attribute values. Each equivalence class has exactly $S$ tuples, which contain sensitive attribute values $S_{1}, \ldots, S_{s}$.

- A probability attribute. The probability attribute of the tuple with $A_{l} \geq v_{l}, \ldots, A_{m} \geq v_{m}$ and $S$ $=S_{k}$ stores the $\mathrm{P}\left(S_{k} \mid A_{l} \geq v_{l}, \ldots, A_{m} \geq v_{m}\right)$ value computed in Equation 5. Similarly, the values of other conditional probabilities from $\mathrm{P}\left(S_{k} \mid A_{1} \geq v_{1}, \ldots, A_{m-1} \geq v_{m-1}, A_{m}<v_{m}\right)$ to $\mathrm{P}\left(S_{k} \mid A_{l}<v_{1}, \ldots\right.$, $\left.A_{m}<v_{m}\right)$ for $k=1, . ., s$ are also stored in this attribute.

As an example, Table 7 shows $P T_{\text {age, ZIP code }}$, which is a probabilistic table for published tables $T_{\text {age }}$ and $T_{Z I P \text { code }}$ in Table 2. The probabilistic table $P T_{\text {age, ZIP code }}$ contains quasi-identifier attributes "Age" and "ZIP Code", a sensitive attribute "Salary Class", and an attribute storing probability values. The first row of $P T_{\text {age, } Z \text { ZIP code }}$ indicates that given the victim's age $\geq 40$ and the victim's ZIP code $\geq 20 \mathrm{k}$, the conditional probability that the salary class of the victim is "High" is 0.4 .

In practice, an adversary may only be able to obtain access to some of the $n$ published tables and/or may only know that some of the $m$ quasi-identifier attribute values that correspond to the victim. Let $A_{1}, \ldots, A_{m^{\prime}}$ be such quasi-identifier attributes in the published tables that can be accessed by the adversary, where $m^{\prime}<m$. In this case, we cannot use $P T_{I \sim n}$ to represent the information deduced by such an adversary. It is because the adversary does not possess enough information to compute those conditional probabilities in $P T_{I \sim n}$. Instead, we use another probabilistic table that only contains quasi-identifier attributes $A_{1}, \ldots, A_{m}$, a sensitive attribute $S$, and a probability attribute to represent the information deduced by the adversary who ignores the quasi-identifier attributes $A_{m^{\prime}+1}, \ldots, A_{m}$ that he/she is not knowledgeable about. Nevertheless, for preserving privacy, we only need to consider the worst case: the adversary is able to access all $n$ published tables. Hence, we do not need to explicitly construct all possible probabilistic tables.

\section{Privacy Preserving Requirement and Algorithm for Non- Centralized Data Publishing}

In this section, we present the non-centralized version of distinct $l$-diversity and an algorithm for non-centralized PPDP. Section 5.1 proposes how to adopt distinct $l$-diversity as a privacy preserving requirement for non-centralized data publishing. Section 5.2 proposes an algorithm, which is modified from Incognito [13], to generate a published table that satisfies non-centralized distinct $l$-diversity. Section 5.3 proves the correctness of applying the modified version of Incognito to the non-centralized data publishing problem.

\subsection{Non-centralized Distinct $l$-diversity}

Suppose there are $n$ data holders, denoted as $D H_{1}, \ldots$, and $D H_{n}$. Each of them solely owns a private table, which have a common sensitive attribute $S$ with the same domain and store information about the same group of individuals. Let $\mathrm{Pri}_{i}$ be the private table owned by $D H_{i}$ for 1 $\leq i \leq n$. For research or other purpose, a published version of the private table (the published table) is released by the data holders. Let $T_{i}$ be the published table released by $D H_{i}$ for $1 \leq i \leq n$. Each published table contains the common sensitive attribute and some quasi-identifer attributes. Let $A_{1}, \ldots, A_{m}$ be the $m$ quasi-identifier attributes in these $n$ published table. Without loss of generality, we assume that $D H_{n}$ is the last data holder to release its published table $T_{n}$, which contains quasi-identifier attributes $A_{m^{\prime}+1}, \ldots, A_{m}$, where $m^{\prime}<m . D H_{n}$ has to ensure that there are no privacy breaches for releasing $T_{n}$ if it is obtained by an adversary who possesses $T_{1}, \ldots, T_{n-1}$ and the identification information of the individuals in these published tables.

We propose that distinct $l$-diversity [2], which is a privacy preserving requirement for centralized data publishing, can also be used for non-centralized data publishing. In other words, it can be used by $\mathrm{DH}_{n}$ to ensure that releasing $T_{n}$ does not cause privacy breaches. It is explained as follows. Consider an equivalence class in probabilistic table $P T_{1 \sim n}$. The number of tuples having non-zero probability attribute values can be regarded as the number of possible sensitive attribute 
values of the victim deduced by an adversary who possesses the identification information of the victim. It shares the same semantic of the $l$ value in distinct $l$-diversity. Hence, we can define distinct $l$-diversity for non-centralized data publishing under our proposed framework as follows.

Defintion 2. (Distinct $l$-diversity for an equivalence class) An equivalence class in probabilistic table $P T_{l \sim n}$ satisfies distinct $l$-diversity if and only if it has at least $l$ tuples having non-zero probability attribute values.

For example, the four equivalence classes in $P T_{\text {age, ZIP code }}$ (Table 7) satisfy distinct $l$-diversity for $l$ $=3,2,2,1$ (from the top to the bottom).

According to distinct $l$-diversity for centralized data publishing, a published table satisfies distinct $l$-diversity if and only if all of its equivalence classes satisfy distinct $l$-diversity. Here we have the same definition for non-centralized data publishing under our proposed framework. It is formally defined as follows.

Definition 3. (Distinct $l$-diversity for $P T_{l \sim n}$ ) A probabilistic table $P T_{l \sim n}$ satisfies distinct $l$ diversity if and only if all of its equivalence classes satisfy distinct $l$-diversity.

For example, $P T_{\text {age, ZIP code }}$ (Table 7) only satisfies distinct $l$-diversity for $l=1$.

$P T_{I \sim n}$ represents the worst case that an adversary can obtain access to all $n$ published tables and knows the values of all $m$ quasi-identifier attributes of all individuals. According to the definition of distinct $l$-diversity [2], if $P T_{l \sim n}$ satisfies distinct $l$-diversity, we can say that no privacy breaches will occur for such a worst case. However, in practice, other possible cases may happen such that the adversary may only be able to obtain access to $n^{\prime}$ published tables and/or may only know $m^{\prime}$ quasi-identifier values about the victim, where $n^{\prime}<n$ and $m^{\prime}<m$. Theorems 1 and 2 show that if $P T_{I \sim n}$ (the worst case) satisfies distinct $l$-diversity, probabilistic tables for all other possible cases will also satisfy distinct $l$-diversity.

Theorem 1. If the probabilistic table $P T_{1 \sim n}$ that is constructed by the adversary who can obtain access to all $n$ published tables satisfies distinct $l$-diversity, the probabilistic table $P T_{I \sim n^{\prime}}$ that is constructed by the adversary who can only obtain access to $n^{\prime}$ published tables also satisfies distinct $l$-diversity, where $n^{\prime}<n$. (Proof skipped due to page limit)

Theorem 2. If the probabilistic table $P T_{1 \sim n}$ that is constructed by the adversary who possesses the values of all $m$ quasi-identifier attributes of the victim satisfies distinct $l$-diversity, the probabilistic table $P T^{\prime}{ }_{1 \sim n}$ that is constructed by the adversary who only possesses the values of $m^{\prime}$ $<m$ quasi-identifier attributes of the victim also satisfies distinct $l$-diversity. (Proof skipped due to page limit)

Theorems 1 and 2 imply that no privacy breach will occur for all other possible cases if the worst case does not cause privacy breach.

Next, we consider published table $T_{n}$. The reason for data holder $D H_{n}$ to construct $P T_{1 \sim n}$ is to check whether privacy breaches will occur for releasing published table $T_{n}$. We say that no privacy breaches will occur for releasing published table $T_{n}$ if probabilistic table $P T_{1 \sim n}$ satisfy distinct $l$-diversity. It is because according to Definition 3 and Theorems 1 and 2 , if $P T_{l \sim n}$ satisfies distinct $l$-diversity, no privacy breaches will occur for the case that an adversary can obtain access to all/some published tables, which may include $T_{n}$. Hence, no privacy breaches will occur for releasing published table $T_{n}$.

The relationship among $T_{n}, P T_{1 \sim n}$, and (non-centralized) distinct $l$-diversity is formally defined as follows.

Definition 4. (Non-centralized distinct $l$-diversity for $T_{n}$ ) If probabilistic table $P T_{l \sim n}$ satisfies distinct $l$-diversity, we say that $T_{n}$ satisfies non-centralized distinct $l$-diversity. 
International Journal of Database Management Systems ( IJDMS ) Vol.4, No.2, April 2012

Note that in order for $T_{n}$ to satisfy non-centralized distinct $l$-diversity with $l=l$, previous published tables $T_{1}, \ldots, T_{n-l}$ have to also satisfy non-centralized distinct $l$-diversity with $l \geq l^{\prime}$. Otherwise, it is possible that $T_{n}$ cannot satisfy non-centralized distinct $l$-diversity with $l=l^{\prime}$ no matter how $T_{n}$ is generalized.

Adopting distinct $l$-diversity [2] to the problem of non-centralized data publishing allow us to propose an efficient algorithm to generate a published table that satisfies non-centralized distinct $l$-diversity. It is explained as follows. Firstly, as proved in Theorems 1 and 2, we only need to ensure that the worst case will not cause privacy breaches instead of checking all possible cases. Secondly, non-centralized distinct $l$-diversity satisfies the generalization property and the subset property, which are necessary to apply Incognito [13] to generate published tables correctly and efficiently (see Theorems 3 and 4).

\subsection{Algorithm}

Intuitively, a data holder, say $D H_{n}$, can use the following steps (Steps 1 to 3) to generate a published table $T_{n}$ that satisfies non-centralized distinct $l$-diversity (later we will show that Step 2 can be skipped).

- $\quad$ Step 1: Collect $T_{1}, \ldots$, and $T_{n-1}$.

- Step 2: Construct a probabilistic table $P T_{1 \sim(n-1)}$ from $T_{1}, \ldots$, and $T_{n-1}$.

- Step 3: Generate $T_{n}$ from $P r i_{n}$ such that $P T_{I \sim n}$ satisfies non-centralized distinct $l$-diversity.

Step 1 is to collect the related published tables that have been already released by other data holders. Step 2 is to construct a probabilistic table $P T_{I \sim(n-l)}$ from the collected published tables. Step 3 is to generate $T_{n}$ by the modified version of Incognito [13], so that $P T_{1 \sim n}$, which is constructed by combining $T_{n}$ and $P T_{I \sim(n-l)}$, satisfies non-centralized distinct $l$-diversity.

Nevertheless, we figure out how to verify whether $P T_{1 \sim n}$ satisfies non-centralized distinct $l$ diversity without actually constructing $P T_{1 \sim n}$. Hence, we do not need Step 2 to construct $P T_{1 \sim(n-1)}$ neither. To explain how to do so, we first describe the steps (Steps A to C) to construct $P T_{1 \sim n}$ from $P T_{1 \sim(n-1)}$ and $T_{n}$.

- Step A: The first step is to generate all equivalence classes of $P T_{1 \sim n}$. Recall that an equivalence class is a collection of tuples with the same quasi-identifier attribute values. Hence, we can use a set of quasi-identifier attribute values to be the name of an equivalence class. The name of an equivalence class in $P T_{1 \sim n}$ can be obtained by taking the union of the name of an equivalence class in $P T_{I \sim(n-1)}$ and that in $T_{n}$. For example, let $X, Y$, and $Z$ be $\left\{A_{l} \geq v_{l}, \ldots, A_{m^{\prime}} \geq v_{m^{\prime}}\right\}$ in $P T_{1 \sim(n-l)},\left\{A_{m^{\prime}+1} \geq v_{m^{\prime}+1}, \ldots, A_{m} \geq v_{m}\right\}$ in $T_{n}$, and $\left\{A_{l}\right.$ $\left.\geq v_{l}, \ldots, A_{m} \geq v_{m}\right\}$ in $P T_{1 \sim n}$ respectively. $Z$ is obtained by taking the union of $X$ and $Y$. The names of all equivalence classes in $P T_{I \sim n}$ are all possible combinations of the equivalence class names in $P T_{I \sim(n-1)}$ and those in $T_{n}$. In other words, the number of equivalence classes in $P T_{1 \sim n}$ equals to the number of equivalence classes in $P T_{I \sim(n-1)}$ multiplies the number of equivalence classes in $T_{n}$.

- Step B: The second step is to generate sensitive attribute values with non-zero conditional probabilities for all equivalence classes in $P T_{1 \sim n}$. Let $S_{X}, S_{Y}$, and $S_{Z}$ be the set of $S$ values with non-zero conditional probabilities in $X, Y$, and $Z$ respectively. In Equation (5), if all the terms on the right hand side are non-zero, the resulting conditional probability on the left hand side is non-zero. If any term on the right hand side is zero, the resulting conditional probability on the left hand side is zero. Hence, if $Z$ is obtained by taking the union of $X$ and $Y$, we have $S_{Z}=S_{X} \cap S_{Y}$. For example, if $S_{X}=$ \{"High", "Middle" $\}$ and $S_{Y}$ $=\{$ "Middle", "Low" $\}$, we have $S_{Z}=\{$ "Middle" $\}$.

- Step C: The third step is to apply Equation (5) to calculate the non-zero conditional probabilities for each sensitive attribute value generated in the Step B. 
International Journal of Database Management Systems ( IJDMS ) Vol.4, No.2, April 2012

For non-centralized distinct $l$-diversity, we are only interested in the number of different sensitive attribute values with non-zero probabilities in each equivalence classes. We do not care about the names of equivalence classes (obtained by Step A) and the actual values for the non-zero conditional probabilities (obtained by Step C). Hence, to verify whether $P T_{I \sim n}$ satisfies noncentralized distinct $l$-diversity, we can skip Step A and Step C and only perform Step B, which becomes the requirement checking step of the modified version of Incognito in Step 3. The modified checking step counts the number of elements in each intersection of every possible combinations among the set of $S$ values with non-zero conditional probabilities for every equivalence class in $T_{n}$ and that in $P T_{I \sim(n-l)}$, and checks if all such numbers are not less than $l$. To know the set of $S$ values in the equivalence classes in $P T_{1 \sim(n-1)}$, we only need to refer to $T_{1}, \ldots, T_{n-1}$. In other words, there is no need to explicitly construct any probabilistic tables, including $P T_{1 \sim(n-1)}$ and $P T_{1 \sim n}$. We only need to count the number of elements in each intersection of every possible combinations among the set of $S$ values with non-zero conditional probabilities for every equivalence class in $T_{l}, \ldots, T_{n}$, and deduce that $P T_{l \sim n}$ satisfy non-centralized $l$-diversity if and only if all such numbers are not less than $l$. For example, suppose two published tables $T_{1}$ and $T_{2}$ have been published by two data holders and the third data holder is now generating $T_{3}$. Assume that $T_{1}$ has three equivalence classes, each of which has the sensitive attribute sets $S_{I A}, S_{I B}$, and $S_{1 C} ; T_{2}$ has two equivalence classes, each of which has the sensitive attribute sets $S_{2 A}$ and $S_{2 B}$. If $T_{3}$ has two equivalence classes, each of which has the sensitive attribute sets $S_{3 A}$ and $S_{3 B}$. An example is shown in Table 8. Suppose two published tables, namely $T_{1}$ and $T_{2}$, are published by two data holders. Both published tables have the same sensitive attribute $S$ with the same domain. Further suppose that the third data holder wants to publish a published table, say $T_{3}$, with the same $S$ sensitive value with the same domain. The third data holder has to consider 12 sensitive attribute set intersections as shown in Table 8(b). If the size of every intersection is not less than $l$, releasing $T_{3}$ satisfies non-centralized $l$-diversity.

Table 8 . The sensitive attribute set intersections that are considered for releasing $T_{3}$ after $T_{1}$ and $T_{2}$ are published.

\begin{tabular}{|c|c|c|}
\hline Published Table & Equivalence Class & $\boldsymbol{S}$ \\
\hline \multirow{3}{*}{$T_{1}$} & $E C_{1 A}$ & $S_{1 A}$ \\
\cline { 2 - 3 } & $E C_{I B}$ & $S_{I B}$ \\
\cline { 2 - 3 } & $E C_{I C}$ & $S_{I C}$ \\
\hline \multirow{2}{*}{$T_{2}$} & $E C_{2 A}$ & $S_{2 A}$ \\
\cline { 2 - 3 } & $E C_{2 B}$ & $S_{2 B}$ \\
\hline \multirow{2}{*}{$T_{3}$} & $E C_{3 A}$ & $S_{3 A}$ \\
\cline { 2 - 3 } & $E C_{3 B}$ & $S_{3 B}$ \\
\hline
\end{tabular}

(a) Published tables $T_{1}, T_{2}$, and $T_{3}$

\begin{tabular}{|c|}
\hline Sensitive Attribute Set Intersections \\
\hline$S_{I A} \cap S_{2 A} \cap S_{3 A}$ \\
\hline$S_{I A} \cap S_{2 A} \cap S_{3 B}$ \\
\hline$S_{I A} \cap S_{2 B} \cap S_{3 A}$ \\
\hline$S_{I A} \cap S_{2 B} \cap S_{3 B}$ \\
\hline$S_{I B} \cap S_{2 A} \cap S_{3 A}$ \\
\hline$S_{I B} \cap S_{2 A} \cap S_{3 B}$ \\
\hline$S_{I B} \cap S_{2 B} \cap S_{3 A}$ \\
\hline$S_{I B} \cap S_{2 B} \cap S_{3 B}$ \\
\hline$S_{I C} \cap S_{2 A} \cap S_{3 A}$ \\
\hline$S_{I C} \cap S_{2 A} \cap S_{3 B}$ \\
\hline$S_{I C} \cap S_{2 B} \cap S_{3 A}$ \\
\hline$S_{I C} \cap S_{2 B} \cap S_{3 B}$ \\
\hline
\end{tabular}

(b) The sensitive attribute set intersections that are considered

Incognito is still efficient with the aforesaid modification. It is because the modified requirement checking step, which is the only step that we modify on Incognito, is still working on equivalence classes and is not computationally intensive.

\subsection{Theorems}

In this subsection, we prove the correctness of applying the modified version of Incognito to the non-centralized data publishing problem. 
International Journal of Database Management Systems ( IJDMS ) Vol.4, No.2, April 2012

To apply Incognito, the privacy preserving requirement has to satisfy the generalization property and the subset property [13]. Generalization property means that suppose $T_{A}$ and $T_{B}$ are generalized from a private table PriT and $T_{B}$ is more general than $T_{A}$; if $T_{A}$ satisfies a privacy preserving requirement, $T_{B}$ also satisfies the same requirement. Subset property means that suppose $T_{C}$ is generalized from a private table PriT and $T_{D}$ is obtained by removing some quasiidentifier attributes of $T_{C}$; if $T_{C}$ satisfies a privacy preserving requirement, $T_{D}$ will also satisfy the same requirement. Theorems 3 and 4 show that non-centralized distinct $l$-diversity satisfies the generalization property and the subset property.

Theorem 3. Non-centralized distinct $l$-diversity satisfies the generalization property. (Proof skipped due to page limit)

Theorem 4. Non-centralized distinct $l$-diversity satisfies the subset property. (Proof skipped due to page limit)

Theorems 3 and 4 imply that we can apply the modified version of Incognito to generate published table $T_{n}$ such that when $T_{n}$ is considered with $P T_{1 \sim(n-1)}$ together to construct $P T_{1 \sim n}, P T_{1 \sim n}$ satisfies distinct $l$-diversity. By Definition 4 , we say that $T_{n}$ satisfies non-centralized distinct $l$ diversity and releasing $T_{n}$ will not cause privacy breach even if it is considered with other published tables $T_{1}, \ldots$, and $T_{n-1}$.

\section{EXPERIMENTS}

In this section, we present the experiment results. The objective of our experiment is to evaluate whether our non-centralized algorithm is feasible to real applications. The algorithm is feasible if it can generate a published table in a reasonable time from real databases, where each of them contains more quasi-identifier (QI) attributes and a large number of tuples. Section 6.1 presents the settings of the experiment. Section 6.2 presents the metrics measured in the experiment. Section 6.3 presents and discusses the experiment results.

Table 9. Schema of $S A L$.

\begin{tabular}{|c|c|c|c|}
\hline Attribute Type & Attribute & Range & Number of Possible Values \\
\hline \multirow{4}{*}{ QI Attributes } & Age & $16-94$ & 77 \\
\cline { 2 - 4 } & Education & $1-17$ & 14 \\
\cline { 2 - 4 } & Birthplace & $1-710$ & 133 \\
\cline { 2 - 4 } & Occupation & $1-983$ & 509 \\
\cline { 2 - 4 } & Race & $1-9$ & 9 \\
\cline { 2 - 4 } & Work class & $2-10$ & 7 \\
\cline { 2 - 4 } & Marital status & $1-6$ & 6 \\
\hline Sensitive Attribute & Income & $0-49$ & 50 \\
\hline
\end{tabular}

\subsection{Settings}

All experiments were performed on a computer with Intel Core 2 Duo $2.80 \mathrm{GHz}$ CPU and $3.2 \mathrm{~GB}$ RAM running Windows XP and IBM DB2 Express-C. We modify Incognito [13] to become our non-centralized algorithm. To simulate a non-centralized data publishing environment, we divide a real (centralized) database $S A L$ (from http://ipums.org) into a number of (non-centralized) private tables. SAL contains 700k tuples, each of which stores information about an American adult. Table 9 shows the schema of $S A L$ that we use, which has seven QI attributes: age, education, birthplace, occupation, race, work class, and marital status; and a sensitive attribute: income. Each possible value of an attribute is represented by an integer. Table 9 shows the range and the number of possible values for each attribute.

Similar to Incognito, our non-centralized algorithm requires users to define generalization levels for each QI attribute to indicate how to generalize the QI attribute in a published table. In our 
International Journal of Database Management Systems ( IJDMS ) Vol.4, No.2, April 2012

experiments, we define four generalization levels for each QI attribute: level-0 represents no generalization, while level-1, level-2, and level-3 represent the generalization that results in four, two, and one equivalence classes respectively.

The four parameters for our experiments are $l,|Q I|, l_{\text {pre }}$, and $\left|Q I_{p r e}\right|$. They are explained as follows.

- $\quad l$ is the $l$ value for the distinct $l$-diversity requirement, i.e. the number of distinct sensitive values for each equivalence class has to be larger than or equal to $l$.

- $|Q I|$ is the number of QI attributes for the current published table.

- $\quad l_{\text {pre }}$ is the distinct $l$-diversity requirement for the previous published table (if there are multiple previous published tables, here refer to the latest one). Note that it is not necessary for $l_{\text {pre }}$ to be equal to the minimum number of distinct sensitive values for all equivalence classes in the (latest) previous published table.

- $\left|Q I_{\text {pre }}\right|$ is the number of QI attributes in the published tables that have been released by other data holders already (i.e. in the previous published tables) before generating the current published table. If $\left|Q I_{p r e}\right|=0$, it means that the published table is generated when there is no previous published tables. In this case, we can use distinct 1-diversity [2] directly. If $\left|Q I_{\text {pre }}\right|>0$, it means that the published table is generated when there are some previous published tables. In this case, our non-centralized algorithm is used.

Note that we do not need to set the number of previous published tables as a parameter in our experiments. It is because such a number does not directly affect the probabilistic table. Instead, we can tune the $\left|Q I_{\text {pre }}\right|$ parameter to control the effect of the previous published tables in the experiments.

\subsection{Metrics}

We measure the following three metrics in our experiment.

1. Elapsed time for generating the published table given a probabilistic table and a private table.

2. The number of published tables that satisfy the requirement. We call these published tables published table candidates. The published table candidates differ in the generalization levels for each QI attribute.

3. The minimum average generalization level. For each published table, there are a number of QI attributes, and each QI attributes are generalized according to the generalization level. Hence, we can calculate the average generalization level for each published table. Similar to Incognito, our non-centralized algorithm outputs a number of published tables. A user only needs to select one published table among all the published table candidates to release. Usually the one that is least generalized (with the lowest average generalization level) is chosen in order to have the highest data quality to the data miner. Therefore, we are interested in the minimum average generalization level among all these published tables. 
International Journal of Database Management Systems ( IJDMS ) Vol.4, No.2, April 2012

Table 10. Parameters for the four experiment sets.

\begin{tabular}{|c|c|c|}
\hline Experiment Set & Parameter & Values \\
\hline \multirow{2}{*}{$\mathrm{A}$} & $l_{\text {pre }}$ & 30 \\
\cline { 2 - 3 } & $\left|Q I_{\text {pre }}\right|$ & 2 \\
\hline \multirow{2}{*}{$\mathrm{B}$} & $l_{\text {pre }}$ & 50 \\
\cline { 2 - 3 } & $\left|Q I_{\text {pre }}\right|$ & 2 \\
\hline \multirow{2}{*}{$\mathrm{C}$} & $l_{\text {pre }}$ & 30 \\
\cline { 2 - 3 } & $\left|Q I_{\text {pre }}\right|$ & 4 \\
\hline \multirow{2}{*}{$\mathrm{D}$} & $l_{\text {pre }}$ & 50 \\
\cline { 2 - 3 } & $\left|Q I_{\text {pre }}\right|$ & 4 \\
\hline
\end{tabular}
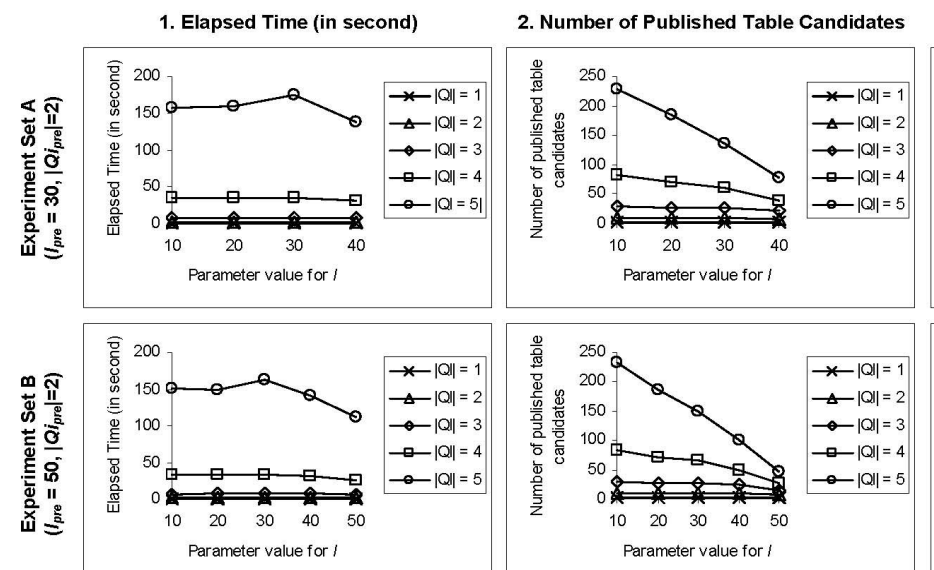

3. Minimum Average Generalization Level
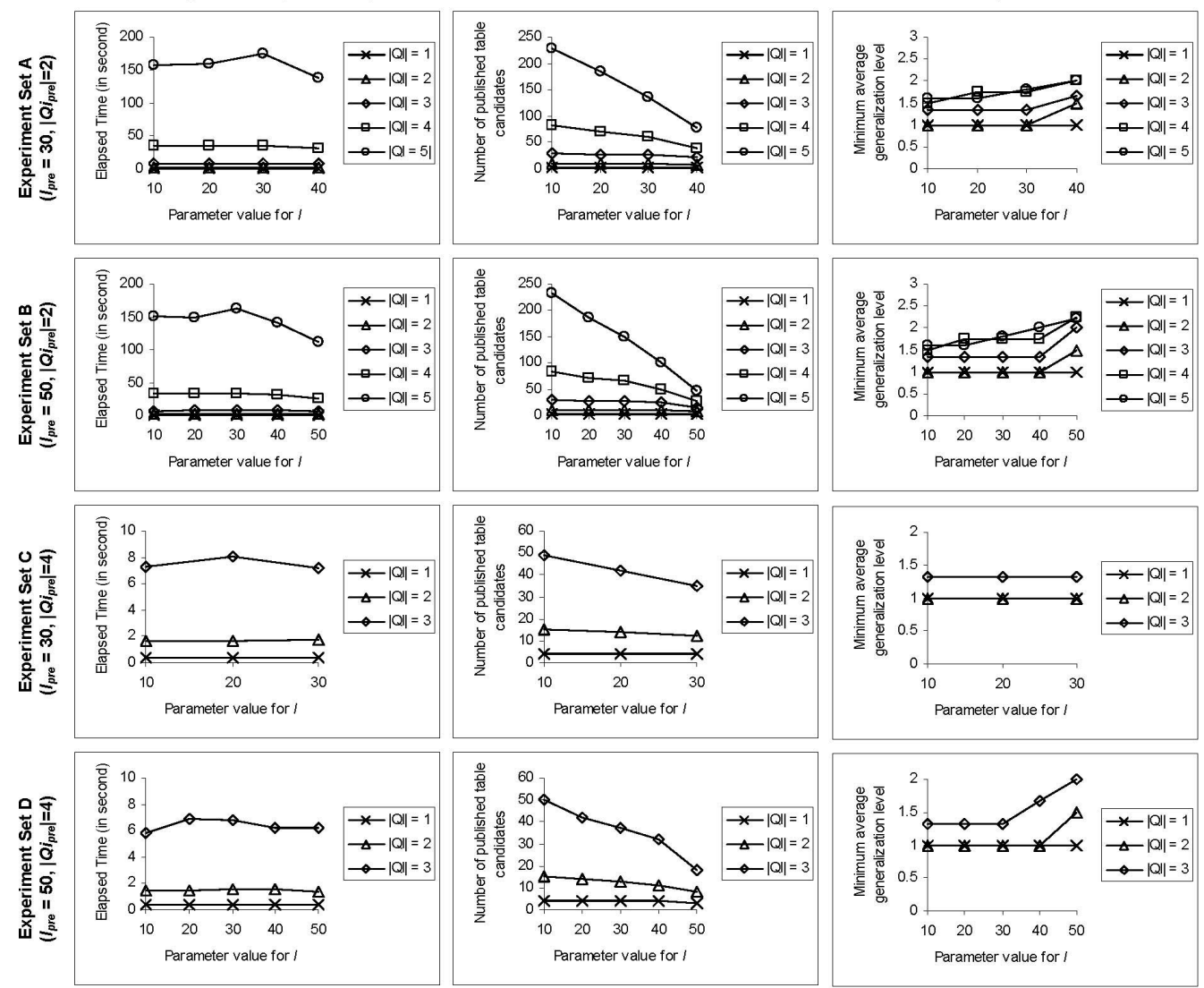

Figure 1. Experimental results.

\subsection{Results and Discussions}

We performed four sets of experiment. Their parameters are shown in Table 10. Figure 1 shows the experimental results. There are three observations about elapsed time based on the experimental results.

1. The first observation is that our non-centralized algorithm takes less than 180 s to generate a published table with five QI attributes.

2. The second observation is that increasing the $\left|Q I_{p r e}\right|$ value does not significantly increase the time to generate a published table. It is because the non-centralized algorithm only reads the sensitive attribute values in each equivalence class in the probabilistic table but 
not the QI attributes. Although the number equivalence classes increases when the $\left|Q I_{\text {pre }}\right|$ value increases, our algorithm only needs to consider one equivalence class among the equivalence classes with the same set of sensitive attribute values. In addition, the time to read the probabilistic table is negligible when comparing with the core algorithm that generates the published table by building and searching for the published table lattice. Hence, the $\left|Q I_{\text {pre }}\right|$ value does not cause much impact to the total elapsed time to generate a published table.

3. The third observation is that our algorithm takes more time to generate a published table with more QI attributes (i.e. a higher $|Q I|$ value), which is reasonable since the lattice size is increased when there are more QI attributes. Therefore, the time needed to build and search the lattice is increased.

The first two observations about elapsed time indicate that our algorithm is feasible for real applications.

There are three observations about the number of published table candidates that satisfy the requirement based on the experimental results. Note that only one of the published table candidates will be chosen as the published table.

1. The first observation is that if the $l$ value increases (i.e. the requirement is more restrictive), fewer published tables will satisfy the requirement. That means the number of published table candidates decreases.

2. The second observation is that if the $|Q I|$ value increases, the number of published table candidates increases. It is because there are more combinations for different generalization levels of QI attributes and hence there are more published table candidates.

3. The third observation is that if the $l_{\text {pre }}$ value increases, the number of published table candidates increases. It is because releasing a new published table can only make the requirement enforced by previous published tables less restrictive. A more restrictive old requirement allows more rooms for it to be lessen and hence more published table candidates can satisfy the new requirement.

There are two observations about the minimum average generalization level based on the experimental results.

1. The first observation is that if the $l$ value increases, then the minimum average generalization level increases. It is because a more generalized published table is needed for a more restrictive requirement.

2. The second observation is that if the $|Q I|$ value increases, then the minimum average generalization level increases. It is because more QI attributes will cause the equivalence classes to have less number of tuples for the same generalization levels. Hence the generalization levels have to be increased in order to satisfy the same requirement.

\section{CONCLUSIONS}

This paper studied the problem of data publishing on multiple non-centralized datasets, which store information about the same group of individuals and share a common sensitive attribute with the same domain. We have shown that such a problem is different from data publishing on a centralized dataset and serial data publishing on a dynamic/static dataset in the sense that there is no dataset that stores all information for non-centralized data publishing. To solve the problem, we first propose a statistical approach, which does not rely on any communications between the data holders, to estimate the information that can be deduced by an adversary who can obtain access to all non-centralized published tables. Then, we propose how to adopt distinct $l$-diversity [2] to non-centralized data publishing. After that, we propose an algorithm to generate published tables that satisfy non-centralized distinct $l$-diversity. Our algorithm is modified from Incognito [13], which is an efficient and widely-used algorithm for (centralized) data publishing. Finally, 
International Journal of Database Management Systems ( IJDMS ) Vol.4, No.2, April 2012

theorems and experiments are presented to show that the proposed algorithm is correct and feasible to real applications.

\section{REFERENCES}

[1] L. Sweeney, (2002) "Achieving $k$-anonymity privacy protection using generalization and suppression”, International Journal on Uncertainty, Fuzziness and Knowledge-based Systems, Vol.10, No.5, pp.571588 .

[2] A. Machanavajjhala, J. Gehrke, D. Kifer, and M. Venkitasubramaniam, (2006) "l-diversity: privacy beyond $k$-anonymity", in proceedings of $I C D E$.

[3] N. Li, T. Li, and S. Venkatasubramanian, (2007) " $t$-closeness: privacy beyond $k$-anonymity and $l$ diversity", in proceedings of ICDE, pp.106-115.

[4] J. Byun, Y. Sohn, E. Bertino, and N. Li, (2006) "Secure anonymization for incremental datasets", in proceedings of $S D M$.

[5] B.C.M. Fung, K. Wang, A. Fu, and J. Pei, (2008) "Anonymity of continuous data publishing", in proceedings of $E D B T$.

[6] X. Xiao and Y. Tao, (2007) “ $m$-invariance: towards privacy preserving re-publication of dynamic datasets", in proceedings of SIGMOD.

[7] Y. Bu, A. Fu, R. Wong, L. Chen, and J. Li, (2008) "Privacy preserving serial data publishing by role composition", in proceedings of $V L D B$.

[8] R.C.W. Wong, A. Fu, J. Liu, K. Wang, and Y. Xu, (2010) "Global Privacy Guarantee in Serial Data Publishing", in proceedings of $I C D E$.

[9] K. Wang and B.C.M. Fung, (2006) "Anonymizing sequential releases", in proceedings of KDD.

[10] P. Andritsos, A. Fuxman, and R. J. Miller, (2006) "Clean answers over dirty databases: A probabilistic approach", in proceedings of $I C D E$.

[11]N. Dalvi and D. Suciu, (2004) "Efficient query evaluation on probabilistic databases", in proceedings of $V L D B$.

[12] R. Cheng, S. Singh, and S. Prabhakar, (2005) "U-DBMS: A database system for managing constantlyevolving data", in proceedings of $V L D B$.

[13] K. LeFevre, D. Dewitt, and R. Ramakrishnan, (2005) "Incognito: efficient fulldomain k-anonymity”, in proceedings of SIGMOD.

[14] R. Agrawal, R. Srikant, and D. Thomas, (2005) "Privacy preserving OLAP", in proceedings of SIGMOD.

[15] S. Zhong, Z. Yang, and R. N. Wright, (2005) "Privacy-enhancing $k$-anonymization of customer data", in proceedings of PODS.

[16] R.J. Bayardo and R. Agrawal, (2005) "Data privacy through optimal $k$-anonymization", in proceedings of $I C D E$.

[17] C. Wang, S. Parthasarathy, and R. Jin, (2006) "A decomposition-based probabilistic framework for estimating the selectivity of XML twig queries", in proceedings of $E D B T$.

[18] S. Chaudhuri, G. Das, V. Hristidis, and G. Weikum, (2004) "Probabilistic ranking of database query results", in proceedings of $V L D B$.

[19] V. Markl, N. Megiddo, M. Kutsch, T.M. Tran, P. Haas, and U. Srivastava, (2005) "Consistently estimating the selectivity of conjuncts of prediciates", in proceedings of $V L D B$.

[20] A. Darwiche, (1997) "A logical notion of conditional independence: properties and applications", Artificial Intelligence, vol. 97, pp. 45-82.

[21]M.G. Walker and G. Wiederhold, (1990) "Acquisition and validation of knowledge from data", Intelligent Systems, chapter 17, pp 415-428. 
International Journal of Database Management Systems ( IJDMS ) Vol.4, No.2, April 2012

[22] G. Shafer, (1998) "Advances in the understanding and use of conditional independence", Annals of Mathematics and Artificial Intelligence, 21.

[23] B.P. Carlin and T.A. Louis, (2008) Bayesian Methods for Data Analysis, Chapman and Hall/CRC.

[24] R. Agrawal and R. Srikant, (2000) "Privacy preserving data mining", in proceedings of SIGMOD.

[25] S. Vijayarani and A. Tamilarasi, (2010) "Bit Transformation Perturbative Masking Technique for Protecting Sensitive Information In Privacy Preserving Data Mining”, International Journal of Database Management Systems (IJDMS).

[26] A. Sharma ${ }^{1}$ and V. Ojha, (2010) "Implementation of Cryptography for Privacy Preserving Data Mining”, International Journal of Database Management Systems (IJDMS). 